\title{
We read spam a lot: prospective cohort study of unsolicited and unwanted academic invitations
}

\author{
Andrew Grey, ${ }^{1}$ Mark J Bolland, ${ }^{1}$ Nicola Dalbeth, ${ }^{1}$ Greg Gamble,, Lynn Sadler
}

Department of Medicine, University of Auckland, Private Bag 92019, Auckland, New Zealand

2Women's Health, Auckland District Health Board, New Zealand

Correspondence to: A Grey a.grey@auckland.ac.nz Cite this as: BMJ 2016;355:15383 http://dx.doi.org/10.1136/bmj.i5383 Accepted: 28 September 2016

\section{ABSTRACT \\ OBJECTIVES}

To assess the amount, relevance, content, and suppressibility of academic electronic spam invitations to attend conferences or submit manuscripts.

DESIGN

Prospective cohort study.

SETTING

Email accounts of participating academics.

\section{PARTICIPANTS}

Five intrepid academics and a great many publishers, editors, and conference organisers.

\section{INTERVENTION}

Unsubscribing from sender's distribution lists.

\section{MAIN OUTCOME MEASURES}

Number of spam invitations received before, immediately after, and one year after unsubscribing from senders' distribution lists. The proportion of duplicate invitations was also assessed and the relevance of each invitation graded to the recipient's research interests. A qualitative assessment of the content of spam invitations was conducted.

\section{RESULTS}

At baseline, recipients received an average of 312 spam invitations each month. Unsubscribing reduced the frequency of the invitations by $39 \%$ after one month but by only $19 \%$ after one year. Overall, $16 \%$ of spam invitations were duplicates and $83 \%$ had little or no relevance to the recipients' research interests. Spam invitations were characterised by inventive language, flattery, and exuberance, and they were sometimes baffling and amusing.

\section{CONCLUSIONS}

Academic spam is common, repetitive, often irrelevant, and difficult to avoid or prevent.

\section{Introduction}

Unsolicited and unwanted (spam) electronic invitations to speak at or attend conferences, or to write for or edit journals are a burgeoning aspect of academic life. Colleagues regard such invitations with wry amusement, intense

\section{WHAT IS ALREADY KNOWN ON THIS TOPIC}

Anecdotal and limited published evidence suggests that spam academic invitations to publish or present research might be common and irritating

\section{WHAT THIS STUDY ADDS}

Academic spam invitations are common and irritating, with 2.1 invitations received daily by each investigator

The incidence of spam invitations is modestly reduced in the first month after unsubscription but the effect wanes after one year

$16 \%$ of spam invitations were duplicates and $83 \%$ were of little relevance to the recipient frustration, or resignation. Two of us (AG, ND) have reviewed travel grant applications from colleagues who received spam invitations to give conference presentations.

Few studies have focused on academic spam. In the Academic Spam Study we investigated the amount, relevance, content, and suppressibility of academic spam emails.

\section{Methods \\ Academic participants}

In a deftly ironic twist, AG emailed five prospective collaborators to invite them to participate in the study. Invitations addressed the recipient as "Eminent Professor," included five or more exclamation marks, and lacked an option to unsubscribe. Non-response to an invitation prompted a flurry of follow-up emails. Inclusion criteria were personal acquaintance with the first author, a sense of humour, a relentless wish to conduct leading edge research, desperation for academic outputs, and an inability to say "no." The exclusion criterion was application of a personal email spam filter. Four of the invited academics agreed to participate; one invitee lacked the inability to say "no."

Collation and analysis of spam and non-spam emails We defined academic spam as unsolicited and unwanted email invitations to attend or present at a conference or to write or edit for a journal. We included all emails the recipients considered to be spam. The investigators collected spam emails received between 1 February 2014 and 30 April 2014. During May 2014, the investigators unsubscribed from the mailing lists of organisations distributing spam. During June 2014 and April 2015, the investigators again collected spam emails.

We assessed the number of spam emails received in each collection phase. Detailed analysis was undertaken of spam received in April 2014, June 2014, and April 2015. The investigators rated their spam invitations as being of no, low, medium, or high relevance to their academic careers. We determined the number of duplicate spam invitations. When possible, we recorded the publisher for journal invitations and organising body for conference invitations. Finally, we conducted a qualitative analysis, focusing on memorable spam.

Between 1 February 2014 and 30 April 2014, the investigators collated invitations to speak at academic meetings or write for journals that they did not regard as spam.

\section{Patient involvement}

No patients were involved in setting the research question or the outcome measures, nor were they involved in developing plans for design or implementation of the study. No patients were asked to advise on interpretation or writing up of results. There are no plans to disseminate the results of the research to study participants or the relevant patient community. 


\begin{tabular}{|c|c|c|c|c|c|c|}
\hline Investigator & Primary training & $\begin{array}{l}\text { Research } \\
\text { experience } \\
\text { (years)* }\end{array}$ & $\begin{array}{l}\text { Publications } \\
\text { at end of } 2013\end{array}$ & $\begin{array}{l}\text { Publications } \\
\text { in } 2013\end{array}$ & $\begin{array}{l}\text { Presentations } \\
\text { in } 2013\end{array}$ & $\begin{array}{l}\text { Height of } \\
\text { esteemt }\end{array}$ \\
\hline$A G$ & Endocrinology & 22 & 210 & 24 & 2 & High \\
\hline MJB & Endocrinology & 10 & 160 & 29 & 2 & High \\
\hline ND & Rheumatology & 11 & 163 & 31 & 8 & High \\
\hline LS & Women's health/epidemiology & 23 & 52 & 6 & NR & High \\
\hline
\end{tabular}

\section{Results}

The Academic Spam Study investigators are mid-career, modestly productive, and conduct research across several disciplines (table 1). On the basis of salutations contained in emails received during the study period, each investigator is highly esteemed. Modesty precludes a systematic description of the height of the esteem but according to these emails each investigator has "made important contributions," is a "distinguished expert," and has "great expertise," sometimes in disciplines surprisingly remote from the primary academic focus.

\section{Bulk spam}

Unsurprisingly, therefore, we received many spam invitations. Between 1 February 2014 and 30 April 2014, 936 spam invitations were received: an average of 312 for each calendar month (fig 1). Spam invitations outnumbered non-spam invitations ( $\mathrm{n}=11$ ) during this period by more than 80 -fold. After unsubscribing from the mailing lists of organisations distributing spam during May 2014, the number of invitations received in June 2014 decreased by 39\%, to 190. In April 2015, the number of spam invitations had increased to 253.

The proportions of spam invitations to write manuscripts and attend conferences were similar before and after unsubscribing (fig 2). Consistently, more than 75\% of spam invitations were of no or low relevance to the recipient (fig 2).

\section{Reheated spam}

In April 2014, June 2014, and April 2015, 74 of 356 (21\%), 30 of 190 (16\%), and 27 of 253 (11\%) of spam emails, respectively, were duplicates. The higher proportion in April 2014 was attributable to the receipt by one investigator of 29 duplicate messages from the Korean Society for Bone and Mineral Research, 19 of which arrived within 72 seconds on one frenetic evening and the other 10 within 57 seconds on a different evening.

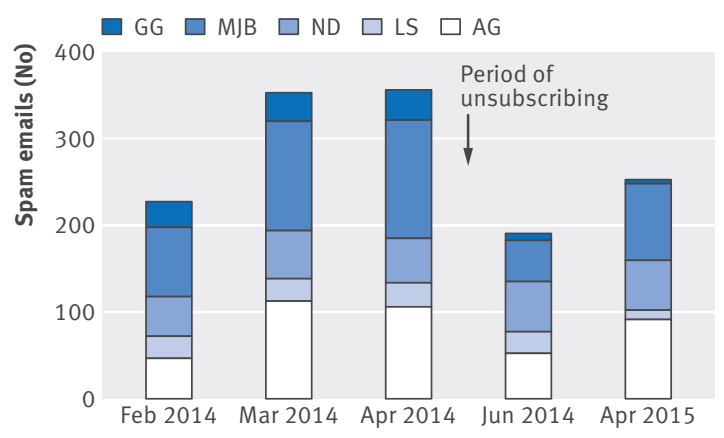

\section{Spam distributors}

During April 2014, four publishers each distributed more than 10 spam invitations to write a manuscript or edit a journal (table 2). These publishers have previously been labelled "predatory."1 Three-Bentham Science, Herbert Publications, and Science Domainprovided an option to unsubscribe. Spam emails from Bentham Science and Herbert Publications almost completely ceased after the month of unsubscribing. For Science Domain, the number of spam emails changed little in the month after unsubscribing, but by April 2015 they had ceased. One publisher, OMICS Group, did not provide an option to unsubscribe and spam email remained substantial in subsequent months. Jacobs Publishers and Open Access Publications sent few spam messages in 2014 but each was a prominent source in April 2015. Eighteen sources of journal related spam were noted in April 2015 that had not featured in April 2014.

Three spam sources sent more than 10 conference invitations in April 2014. Two, BIT Life Sciences and OMICS Group, did not offer unsubscription and remained prominent sources of spam related to conferences a year later.

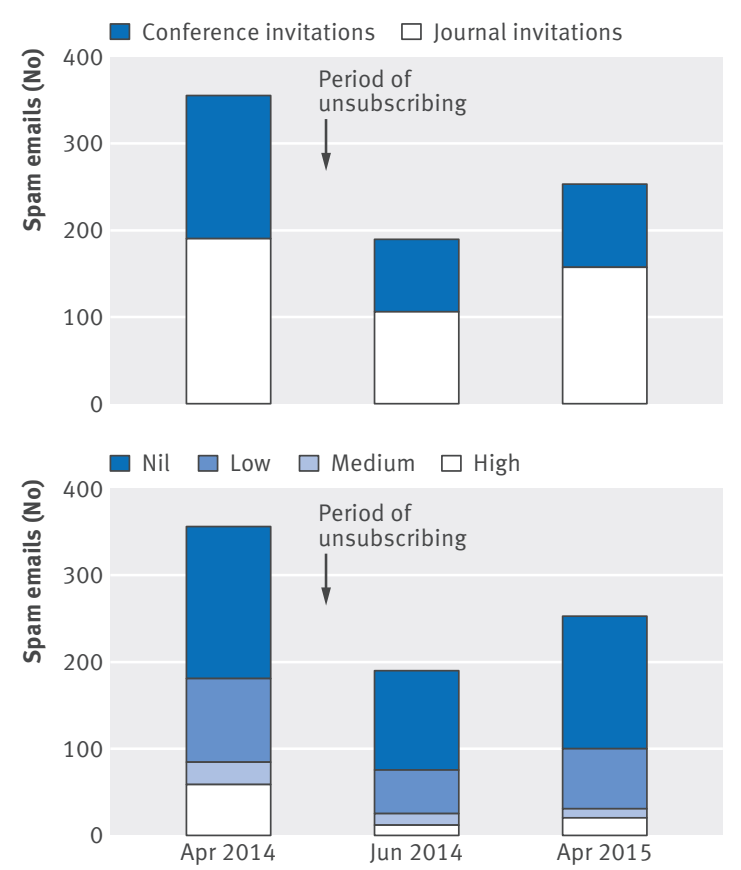

Fig 2 | Number of spam invitations by type and relevance to recipient
Fig 1 Number of spam month of sampling. Stacked bars indicate individual investigators 
Table 2 | Academic spam invitations from sources generating more than 10 invitations during at least one month

\begin{tabular}{llll}
$\begin{array}{l}\text { Sources of spam } \\
\text { Publishers: }\end{array}$ & April 2014 & June 2014 & April 2015 \\
\hline Bentham Science & 18 & 0 & 0 \\
\hline Herbert Publishing & 30 & 1 & 0 \\
\hline Jacobs Publishers & 0 & 0 & 16 \\
\hline OMICS Group & 32 & 27 & 17 \\
\hline Open Access Publications & 5 & 0 & 12 \\
\hline Science Domain & 17 & 13 & 0 \\
\hline Conference organisers: & & & \\
\hline BIT Life Sciences & 12 & 10 & 8 \\
\hline Korean Society for Bone and Mineral Research & 29 & 0 & 0 \\
\hline OMICS Group & 13 & 19 & 7
\end{tabular}

\section{Spam dressed as lamb}

We identified some eye catching subject lines. We learnt that good times happen at oral health conferences ("Learn and Have Fun at the International Conference on Orthognathic Surgery and Orthodontics"). We were tempted by "Cracking the Mysterious Psychiatric Disorders at Euro Psychiatry 2015," and to "Unleash (y)our research ideas at Orthopedics and Rheumatology 2014." We were interested in "Biologically signifying the clinical molecule," and extremely interested in "Special Issue on Wine Health"-sadly, no offers to recruit mid-career academics to studies of wine were apparent. If we had a Startup, we would certainly want to know the answer to the question, "Think your Startup has the WOW factor?"

\section{Tasty spam}

We found several memorable examples of tasty academic spam (box 1). We were impressed by the great enthusiasm of the invitations, featuring up to six exclamation marks! Flattery and inventive turns of phrase were prominent. Some invitations were unrestrainedly aspirational, and some conferences sported intriguing themes. We barely resisted the lure of an "Honorable certificate from MedCrave Editorial Office for publication of your eminent research in the inaugural issue."

\section{Stir fried spam}

Sometimes we found scrambled spam. We were unsure what to make of the session at the 3rd Annual World Congress of Geriatrics and Gerontology 2015 that "can provide you with a best promotion channels in partner seeking, investor relation retreatment and talent search." Or of the message from the International Journal of Applied Biology and Pharmaceutical Technology that invited contribution of a manuscript for publication in the International Journal of Plant Animal and Environmental Science. We were bemused that "the Jacobs Journal of Experimental Dermatology (JJED), invites experts in addiction research to submit original ..." Rather too many invitations were disrespectful of our eminent selves-for example,

\section{Box 1: Examples of tasty academic spam}

\section{Friendly and exuberant}

"We would be really happy to anchor with you"

"Let your wisdom enkindle others"

"Looking forward for an everlasting scientific relationship!"

"I would like to extend my sincere congratulations on the publication of your highly cited original article, 〈Prevalence and clinical factors associated with gout in patients with diabetes and prediabetes, > in the field of diabetes. As of today, this article has been cited more than $\underline{4}$ times"

"It gives us immense pleasure to share this moment of happiness that Journal of Global Economics is planning to release continuous issues every month" "The purpose of this letter is to solicit your gracious presence as a speaker ..."

"We have been through your articles and we are enthralled to know about your reputation and commitment in the field"

"We have chosen selective scientists who have enormously contributed to the scientific community to have their work publish in our journal"

\section{Aspirational and dedicated}

"Aspire to clear all the barriers in dissemination of information and knowledge around the world"

"We aim to enlighten the lamp of information across the sphere especially in the areas of science and technologies"

"Ommega Publishers welcomes you to the newest chapter in the long history of scientific manuscript publication"

"International Journal of Cardiovascular Research, a new frontier among the peer-reviewed scholarly Journals ..."

"GBC 2015 will ... draw together both novice and veterans from the biotechnological front from all over the world to herald avenues to innovations and advancements in the biotechnology sphere both at regional and global level”

"We are creating a kind of mind storming forum to create a new therapeutic approaches"

"Our dedicated proofreaders, cheerfully labor on your manuscripts in a speedy way, with high quality standards on the back of their minds and offer you very appropriate content improvisation wherever required"

"The scientific program paves a way to gather visionaries through the research talks and presentations and put forward many thought provoking strategies"

"Hence the need for integrating the research into the fast paced era needs the a source of rapid dissimilation with a reliable platform. We invite you to be a part of this modern perception by going open access with us"

\section{Thematic}

Lipids 2015- "Solving the impetus of innovations in lipid world"

World Congress of Oral and Dental Medicine- "Keep the door of lives"

The 7th Annual International Congress of Cardiology_ “Bring new vitality into life"

*Emphasis is ours. 
Box 2: Premium spam, featuring intriguing journals and conferences we wish we had attended

\section{Journals}

- International Journal of Advances in Case Reports

- Hair: Therapy and Transplantation

- Therapeutic Hypothermia and Temperature Management

- Journal of Investigative Medicine High Impact Case Reports

- Journal of Ancient Diseases and Preventive Remedies

- Journal of Laboratory Automation

- Interdisciplinary Journal of Microinflammation

\section{Conferences}

- Friends of Israel Urological Symposium 2014

- Global 1000: Meet | Partner | Deal: Showcase + Conference Sept 2014

- Conference of the Global Innovation and Knowledge Academy (GIKA): “Turning Kurt Lewin on his head: Nothing is so theoretical as a good practice"

- World Congress on Controversies in Bovine Health, Industry and Economics

- 2015 International Conference on Steel and Composite Structures

"Dear Dr. MJ Mark JMJ Mark J,” “Dear Dr. Name, Greetings for the day!," and "Dear Dr. \{firstname\}."

\section{Premium spam}

Some spam was almost too delicious to ignore. Box 2 lists journals and conferences that we found especially intriguing.

\section{Discussion}

The Academic Spam Study shows that mid-career academics in New Zealand receive on average 2.1 spam invitations each day to publish papers and attend conferences. Unsubscribing had a modest and short lived effect on the quantity of received spam. Sixteen per cent of spam invitations were duplicates, and $83 \%$ were of little or no relevance to the recipient. Some organisations send spam invitations without an unsubscribe option, or persist despite recipients requesting unsubscription.

\section{Strengths and weaknesses of this study}

Our study has limitations. Some invitations were removed by the institutional spam filter, so we might have underestimated the amount of spam. Our sample of researchers was too small to be representative of the academic community. New Zealand is a small, remote country that might not be targeted by academic spam distributors, even though we have held the Rugby World Cup since 2011, and the Lord of the Rings movies were filmed here. We received a similar number of spam invitations to colleagues in Poland and Canada who assessed journal invitations, however. ${ }^{23}$

\section{Comparison with other studies}

Published research on academic spam is limited. Some senders of spam journal invitations are bad eggs, ${ }^{4}$ who misrepresent their locations and are usually open access publishers. ${ }^{25}$ Spam invitations are often issued by predatory organisations, ${ }^{23}$ the modus operandi of which threatens academic integrity. ${ }^{56}$ Vigorous responses to spam invitations might generate humorous outcomes but not stop the invitations. ${ }^{7}$ Attempts to unsubscribe from spam invitations are only moderately successful, but stringent email filtering ${ }^{3}$ or threatening recidivist organisations with legal action ${ }^{8}$ might stop further communications.

\section{Implications and future research}

We suggest further research on academic spam:

"Nobel and prestigious colleagues,

We are enthralled by prospect of novel research focus of academic spam so we make a proposition to improve enlightenment of evidence. We wish greatly to start journal and convene scientific meeting that focus on academic spam, so illustrious colleagues can form interdisciplinary web of scientific rigour to advance knowledge. Maybe we will christen soon Journal of Advances in Interdisciplinary Academic Spam and launch with alacrity the First Annual International Symposium on Academic Spam (Spam-2017). Once we identify publisher and conference organiser we will email academics to join this exciting novel venture! Honourable colleagues, stay tuned!!!!!!”

Contributors: AG conceived the study, wrote the draft protocol, collated and analysed the data, and wrote the first draft of the paper. He is the guarantor. MIB, ND, GG, and LS refined the protocol, collected the data, and critically reviewed the draft manuscript. All authors had access to the full dataset, take responsibility for the integrity of the data and the accuracy of the dataset, and gave final approval for the submission of this version for consideration of publication.

Funding: This study received no external funding.

Competing interests: All authors have completed the ICMJE unified disclosure form competing interest form at www.icmje.org/coi disclosure.pdf (available on request from the corresponding author) and declare: no support from any organisation for the submitted work no financial relationships with any organisations that might have an interest in the submitted work in the previous three years. Relevant non-financial interests include: AG recently helped his son study persuasive language techniques for a high school term test. MJB uses exclamation marks in emails to indicate disbelief or for ironic emphasis, with the number of exclamation marks proportional to the lack of credibility of the statement. He dislikes exclamation marks used for other reasons. ND almost joined the editorial board of Hair: Therapy and Transplantation but decided her hair was fabulous enough. GG is developing the Number Needed to Spam, an index of how many recipients need to be spammed to recruit one conference attendee. LS wishes henceforth to be addressed solely as Iconic Professor. Ethical approval: Not required.

Data sharing: Data available for academic researchers on request. Transparency: The lead author (AG) affirms that the manuscript is an honest, accurate, and transparent account of the study being reported; that no important aspects of the study have been omitted; and that any discrepancies from the study as planned have been explained.

This is an Open Access article distributed in accordance with the Creative Commons Attribution Non Commercial (CC BY-NC 3.0) license, which permits others to distribute, remix, adapt, build upon this work non-commercially, and license their derivative works on different terms, provided the original work is properly cited and the use is noncommercial. See: http://creativecommons.org/licenses/by-nc/3.0/.

1 Scholarly Open Access. Beall's List. Potential, possible, o probable predatory scholarly open-access publishers. https:// scholarlyoa com/publishers/.

2 Kozak M, lefremova O, Hartley J. Spamming in scholarly publishing: A case study. J Assoc Info Sci Tech, 2015, 10.1002/asi.23521.

Moher D, Srivastava A. You are invited to submit.... BMC Med 2015:13:180. doi:10.1186/s12916-015-0423-3.

4 The Free Dictionary. Bad egg. http://idioms.thefreedictionary.com/bad+egg Moher D, Moher E. Stop predatory publishers now: act collaboratively. Ann Intern Med 2016:164:616-7. doi:10.7326/M15-3015.

6 Butler D. Investigating journals: The dark side of publishing. Nature 2013;495:433-5. doi:10.1038/495433a.

$7 \quad$ Scholarly Open Access. Bogus journal accepts profanity-laced anti-spam paper. https://scholarlyoa.com/2014/11/20/ bogus-journal-accepts-profanity-laced-anti-spam-paper/.

8 Mazzarello S, Fralick M, Clemons M. A simple approach for eliminating spam. Curr Oncol 2015·23.75-6. doi:10.3747/co.23.2860. 